\title{
Investigation of Microstructure and Mechanical Properties of AlTiCrN, AlCrN Coatings Deposited by Advance Sputtering Technique
}

\author{
A. Kulkarni ${ }^{1}$, G. Joshi ${ }^{2}$, N. Patil ${ }^{3}$, V. Sargade ${ }^{4}$ \\ ${ }^{1}$ Associate Professor,VIIT, Pune, India \\ ${ }^{2}$ Assistant Professor, MPSTME, Mumbai, India \\ ${ }^{3}$ Professor, MIT, Aurangabad, India \\ ${ }^{4}$ Professor, DBATU, Lonere, India \\ \{atul.kulkarni@viit.ac.in\}
}

\begin{abstract}
Developments in PVD coating technique for cutting tools enable high speed and dry machining with superior cutting parameters in commercial manufacturing sectors. The present research work investigate the microstructure and the mechanical properties of the AlTiCrN, AlCrN coatings deposited by High Impulse Pulsed Magnetron Sputtering (HIPMS) and Unbalanced Magnetron Sputtering (UBMS) technique respectively onto fine-grained tungsten carbide (K-10) substrate. The surfaces' morphology and microstructure of the AlTiCrN, AlCrN coatings were observed on the scanning electron microscopy. The thin film structure was tested using X-ray diffraction method. The micro-hardness tests were performed and found to be 36.2 and 32 GPa respectively for each coating. Tests of the coatings' adhesion to the substrate material were made using the scratch tester and critical load observed to be $117 \mathrm{~N}$ and $88 \mathrm{~N}$ for AlTiCrN and AlCrN coating respectively. These mechanical properties are crosschecked by machining performance by evaluating tool life. It is found that the structure of the PVD coatings consisted of fine crystallites, while their average size fitted within the range of 11-25 nm, depending on the coating type. The coatings demonstrated columnar structure and dense crosssection morphology as well as good adhesion to the substrate. The very good mechanical properties of the composite coatings make them suitable in industrial applications. The results will provide useful information with respect to the application of composite coatings for the improvement of mechanical properties for dry machining of stainless steel -difficult to machine material.
\end{abstract}

Keywords: HIPMS, UBMS, Microstructure, AlTiCrN, AlCrN

\section{Introduction}

Surface engineering plays a vital role in enhancing the surface properties of the cutting tool. In the present industrial scenario, coated tools are most commonly used in the machining of different 'difficult to machine' materials. Coatings, tool materials and tool geometries have a profound influence due to the increasing requirements of modern and innovative production processes. Nowadays, CVD and PVD techniques are most commonly use for coating deposition. It improves the mechanical, tribological, cutting properties or other qualities of the coatings. Some ecological issues too are developed due to the CVD process byproducts. Hence, the use of CVD coatings is limited nowadays [1-2]. The focus of tool coating industry is shifting towards PVD process, which is another type of tool coating process. There are two major categories of PVD viz. evaporation and sputtering.

The cathodic arc deposition technique is well-established PVD technique, successfully used mainly for the preparation of hard, wear-resistant coatings for tools and different mechanical parts. It has many good properties, such as high ionization and good homogeneity of coatings on substrates of complex shape. Also, it is used to deposit metallic as well as compound thin films for a wide range of applications. In most cases, it is beneficial to produce dense, defect-free coatings, which often requires low-energy ion bombardment of the condensing film surface to increase the mobility of adatoms to achieve void free thin films [3-4].

B. Iyer, S. Nalbalwar and R. Pawade (Eds.)

ICCASP/ICMMD-2016. Advances in Intelligent Systems Research.

Vol. 137, Pp. 311-315.

(C) 2017. The authors - Published by Atlantis Press

This is an open access article under the CC BY-NC license (http://creativecommons.org/licens)es/by-nc/4.0/).

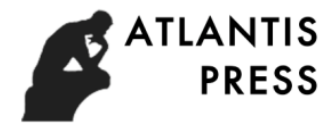


Development of PVD method was caused a large progress in field of tool materials and their wear resistance, mainly tribology. Analyzing properties of coatings developed in the PVD processes one has to pay special attention to issues connected with their mechanical properties, physical properties, abrasive wear resistance, diffusion, and thermal protection, structure, chemical composition and thickness of the coatings [5]. Adhesion of the coating to the substrate features one of the key issues pertaining to coating items with the hard ceramic materials. For dry and hard machining as well as for high cutting speed applications, high hardness and high oxidation resistance at elevated temperatures of more than $700^{\circ} \mathrm{C}$ are of at most importance for the cutting tool [6-7].

Vapor deposited metallic nitride coatings have been widely used to improve the tribological properties of cutting tool substrates. TiN coating is one of the first coatings used successfully to machine steel in industry. (Ti, Al)N coating material was introduced due to its excellent mechanical properties like hardness and thermal stability. It possesses high temperature stability by forming a dense, highly adhesive, protective $\mathrm{Al}_{2} \mathrm{O}_{3}$ surface film in the process of cutting and prevents diffusion of oxygen into the coating. Addition of $\mathrm{Cr}$ in $(\mathrm{Ti}, \mathrm{Al}) \mathrm{N}$ coating further increases the thermal stability by forming $\mathrm{Cr}_{2} \mathrm{O}_{3}$ film on the surface. Also, $\mathrm{CrN}$ sub layer reduces stress and improves adhesion which enhances mechanical properties. $\mathrm{Cr}-\mathrm{Al}-\mathrm{N}$ elements in the hard coatings exhibit considerable oxidation resistance due to the formation of stable and dense $\alpha(\mathrm{Al}, \mathrm{Cr})_{2} \mathrm{O}_{3}$ mixed oxide scales which makes them promising candidates for advanced machining and other high temperature applications [8-9].

The present research work explores the microstructure and the mechanical properties of the AlTiCrN, AlCrN coatings deposited by HIPMS and UBMS technique respectively onto fine-grained tungsten carbide (K-10) substrate.

\section{Experimental Details}

The tests were made on WC-Co cutting inserts (CNMA 120408) deposited by HIPMS technique with AlTiCrN and UBMS with AlCrN hard coatings respectively. Depositions were performed on an industrial deposition system from cemecon, India on their advanced coating System. Micro-hardness is very important parameter which has a major impact on coating. The microhardness tests of coatings were made with the Vicker's hardness tester shown in Fig. 1 (a). The test conditions were selected in order as to be comparable for all coatings. Measurements were made with $25 \mathrm{gf}$ load, to eliminate the substrate influence on the coating hardness. Tests of the coatings' adhesion to the substrate material were made using the scratch test on the SCRATCH TESTER TR -101 shown in Fig. 1(b). In the scratch test, a diamond stylus of $200 \mu \mathrm{m}$ tip radius was drawn over the sample surface under a continuously increasing normal force until the coating detaches. In practice, the film is seldom removed entirely from the channel, so it is convenient to define a critical load (Lc); this being the load at which the coating is removed in a regular way along the whole channel length. Coating detachment may be observed using optical or scanning electron microscopy, and acoustic emission.

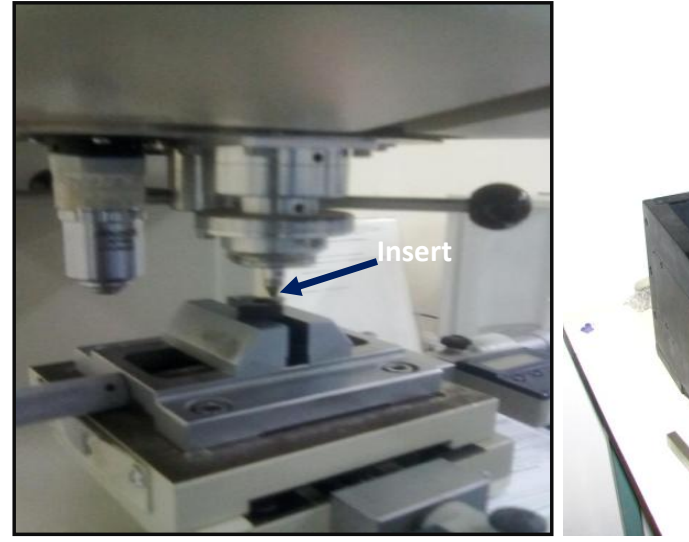

(a)

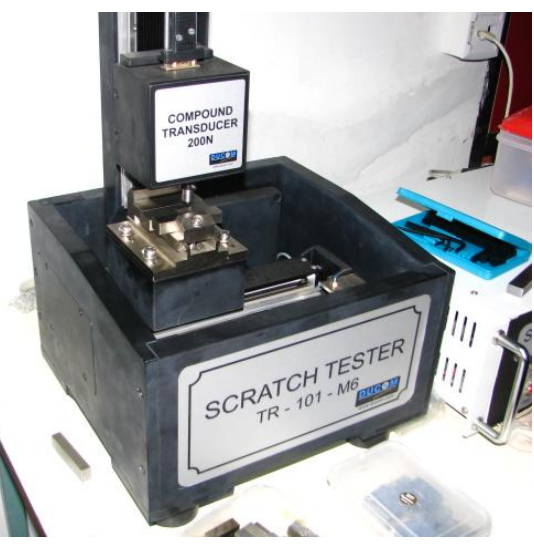

(b)

Fig. 1. (a) Vicker's Micro-Hardness Tester and (b) Photograph of Scratch Tester

Surface morphology, coating microstructure, and coating thickness were studied by observing the coating surface and fractograph under SEM (“JEOL 5800" Japan) and field emission SEM (JEOL, JSM-6700 F). Coating thickness for AlTiCrN and $\mathrm{AlCrN}$ coating is aimed around $3.5 \mu \mathrm{m}$ to $4.5 \mu \mathrm{m}$. It was measured using 
Scanning Electron Microscopy on SEM JEOL 5800 microscope. The coated substrate was cut by wire EDM leaving around 0.5 to $1 \mathrm{~mm}$ thickness to measure coating thickness provided on the tool material and then fracturing it so as to get a clean brittle fracture.

\section{Results and Discussion}

\subsection{Microhardness}

In the present research micro-hardness of AlTiCrN and $\mathrm{AlCrN}$ coating were calculated. The hardness of the coatings tested fits within the range from 32 to $38 \mathrm{GPa}$. The measured micro-hardness of all tools is given in Table 1.

Table 1 Micro-hardness of all Tools

\begin{tabular}{|l|l|l|}
\hline Tool & Micro-hardness \\
\hline AlTiCrN & $3710 \mathrm{HV}$ & $36.3 \mathrm{GPa}$ \\
\hline AlCrN & $3270 \mathrm{HV}$ & $32 \mathrm{GPa}$ \\
\hline
\end{tabular}

\subsection{Scratch Test}

The scratch test was carried out on $\mathrm{AlTiCrN}$ and $\mathrm{AlCrN}$ coated cemented carbide samples using loading rate of $5 \mathrm{~N} / \mathrm{mm}$ and scratch speed of $0.2 \mathrm{~mm} / \mathrm{sec}$. The variation of normal load, traction force and coefficient of friction with the scratch length (stroke) as recorded during the tests are shown for both coatings in Fig. 2 (a) and (b) respectively. This figure also shows the optical photographs of the scratch tracks captured at the location where initiation of coating failure took place. The critical loads of $88 \mathrm{~N}$ and $103 \mathrm{~N}$ are observed for the coating respectively. The critical loads obtained during the present investigation represent the average of at least three different values obtained by performing the scratch test at three different locations on the coated surface. The cumulative specification of the test results are presented in Table 2.

Table 2 Characteristics of the tested coatings

\begin{tabular}{|l|l|l|l|l|}
\hline Coating & Thickness $[\mu \mathrm{m}]$ & Micro-hardness [GPa] & Surface Roughness $[\mu \mathrm{m}]$ & Critical Load LC $[\mathrm{N}]$ \\
\hline AlTiCrN & $\sim 4.5$ & 36.3 & 0.13 & 117 \\
\hline $\mathrm{AlCrN}$ & $\sim 3.6$ & 32 & 0.29 & 88 \\
\hline
\end{tabular}

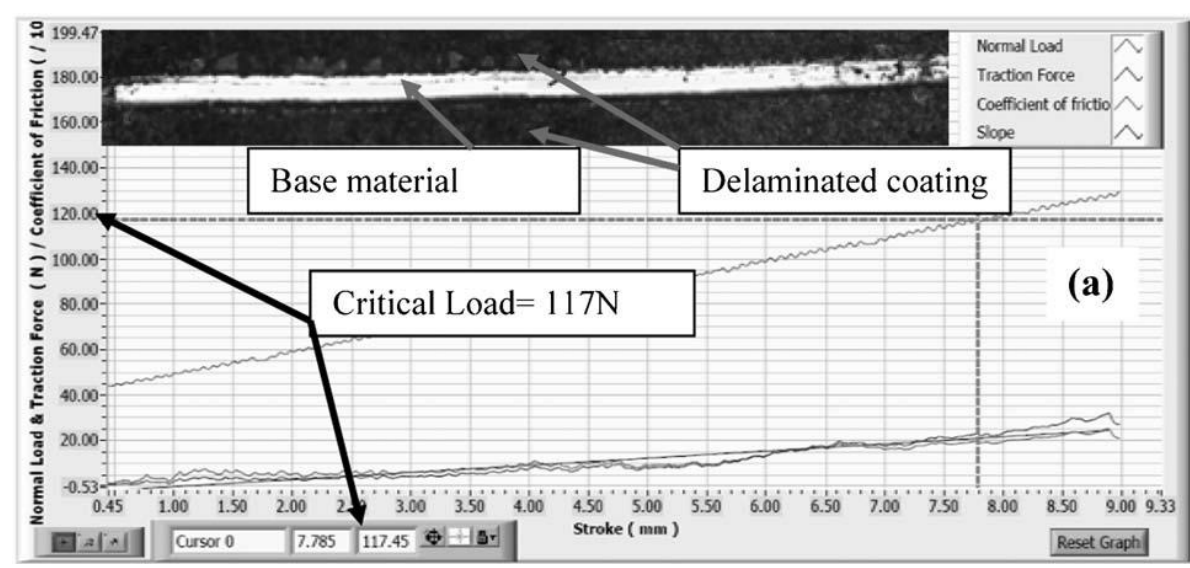




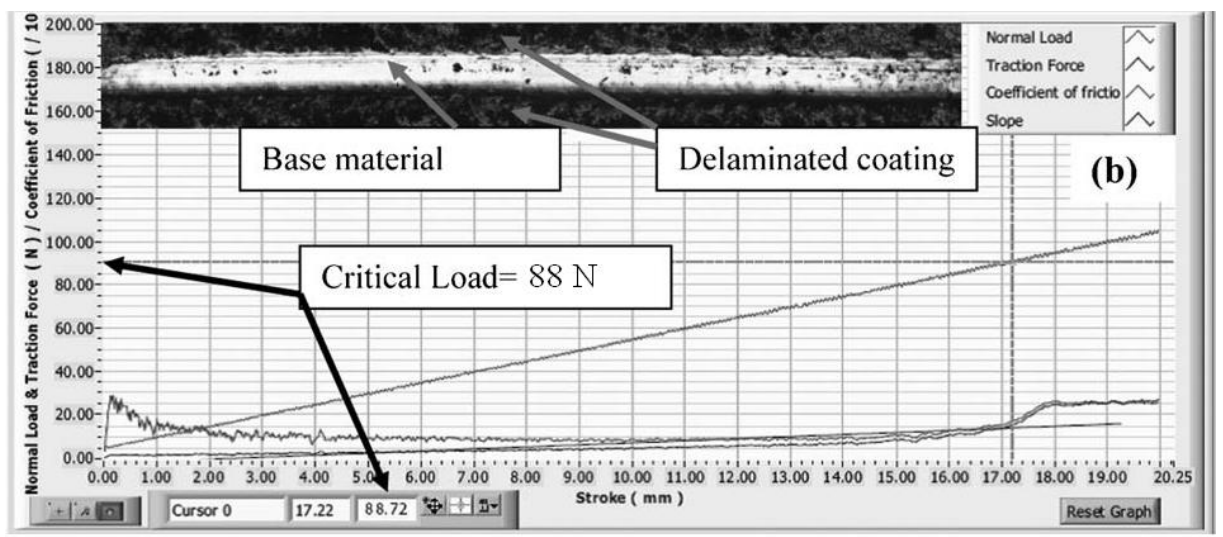

Fig. 2. Critical Load for (a) AlTiCrN and (b) AlCrN coatings

\subsection{Coating Thickness and Surface Morphology}

The coatings present a compact structure, without any visible delaminations or defects. The morphology of the fracture of coatings is characterized with a dense structure, in some cases there is a columnar structure. The fractographic studies of the fractures of the cemented carbide samples examined with the coatings deposited on their surface show a sharp transition zone between the substrate and the coating. SEM fractograph for AlTiCrN, AlCrN coatings are shown in Fig. 3.

The morphology of the coatings' surfaces deposited on the cemented carbide substrate is characterized by a significant in-homogeneity connected with occurrences of the multiple near-drop-shaped or close to spherical particles, which is connected with the essence of the used High Impulse Pulsed Magnetron Sputtering process for depositing the coatings. Apart from single drops one can observe double particles and agglomerates created from several connected particles. The scanning electron microscope (SEM) is used for the analysis of surface topographies and fracture morphology of metallic thin films. Surface topology for AlTiCrN, AlCrN coatings are shown in Fig. 4.
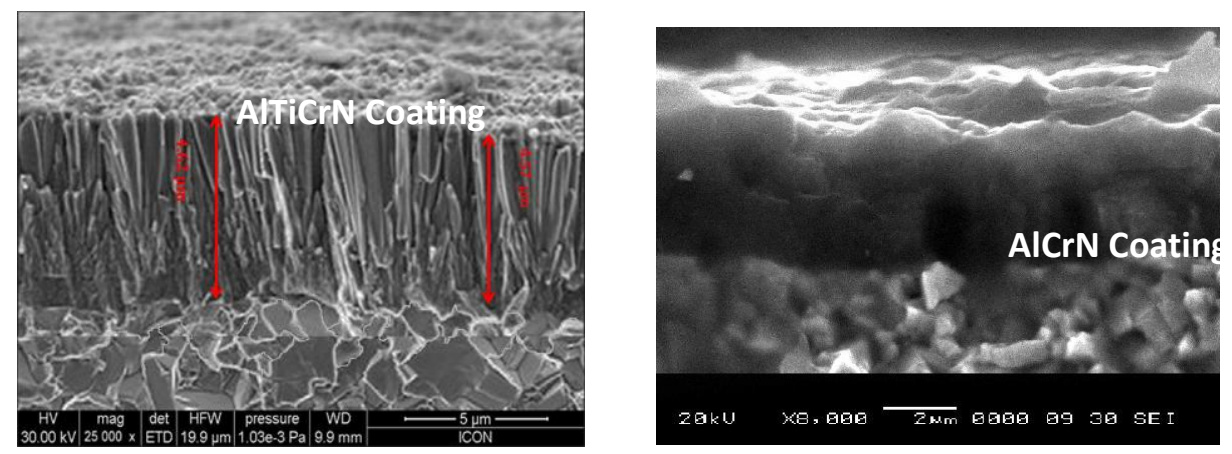

Fig. 3. SEM fractograph for coating thickness of AlTiCrN and AlCrN coating 

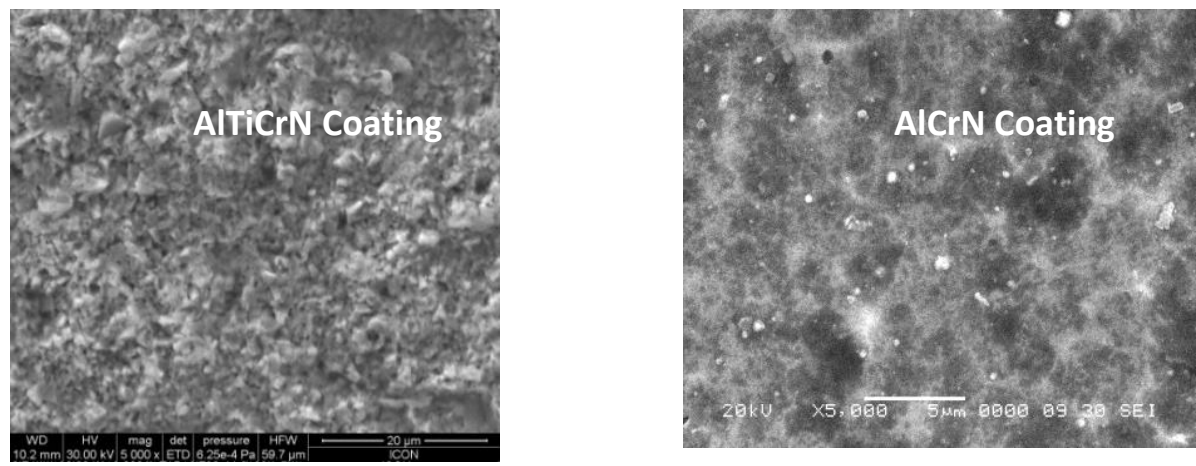

Fig. 4. Surface morphology of AlTiCrN and AlCrN coating

\section{Conclusions}

Coatings of AlTiCrN deposited using HIPMS demonstrated better mechanical properties than coating of $\mathrm{AlCrN}$ deposited using UBMS-PVD technique onto the substrate of tungsten carbide (K-10). The compact structure of the coatings without any visible delaminations was observed.. The fracture morphology of the coatings tested is characterized with a dense and columnar structure. The structure of the coatings consisted of fine crystallites, while their average size fitted within the range of 11-25 $\mathrm{nm}$. The coatings demonstrated columnar structure and dense cross-section morphology as well as good adhesion to the substrate. The critical load LC was found $117 \mathrm{~N}$ and $88 \mathrm{~N}$ for AlTiCrN and AlCrN respectively. The AlTiCrN coating demonstrated a high micro-hardness of 37 GPa whereas AlCrN coating showed micro-hardness of $32 \mathrm{GPa}$ respectively. The above coating can be a potential coating for machining of difficult to cut materials.

\section{References}

[1]. S. PalDey, S.C. Deevi, "Single layer and multilayer wear resistant coatings of (Ti, Al)N: A review", Materials Science and Engineering A,342(2003),58-79

[2]. I. Ciftci, "Machining of austenitic stainless steels using CVD multi-layer coated cemented carbide tools", Tribology International, 39(2006),565-569

[3]. I Petrov, L. Hultman, JE Sundgren, JE Greene,. Journal of Vacuum. Science and Technology,10(2):2 (1992), 65-72

[4]. L. Hultman, U. Helmersson, SA Barnett, JE Sundgren, JE Greene, Journal of , Applied Physics,61(2) (1987), 552-557

[5]. H. Hasegawa, K. Ichijo, T. Suzuki,, Surface and coating Technology, 201(2007), 5477-5480

[6]. D. Rafaja, A. Poklad,, V. Klemm, G. Schreiber, D. Heger, M. Sima, Material Science and Engineering 462(2007), 279-282,

[7]. R.F. Bunshah, Handbook of Hard Coatings, Noyes Publications, New Jersey, USA, 2001

[8]. S.C. Veldhuis , G.K.Dosbaeva , K.Yamamoto, Tribology International, 42 (2009), 1004-1010

[9]. Shin MinLee, HanMingChow, FuangYuanHuang, BiingHwaYan, International Journal of Machine Tools \& Manufacture , 49 (2009), 81-88 